\title{
Clinical Utility of Methylation-Specific Multiplex Ligation-Dependent Probe Amplification for the Diagnosis of Prader-Willi Syndrome and Angelman Syndrome
}

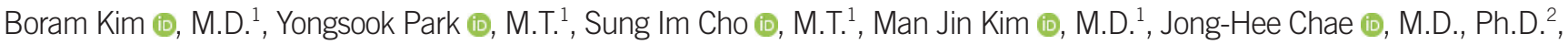

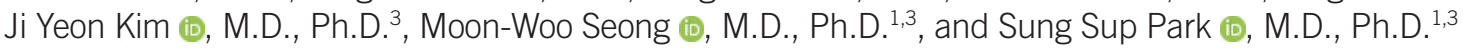

${ }^{1}$ Department of Laboratory Medicine, Seoul National University Hospital, Seoul National University College of Medicine, Seoul, Korea; ${ }^{2}$ Department of Pediatrics, Seoul National University Children's Hospital, Seoul National University College of Medicine, Seoul, Korea; ${ }^{3}$ Biomedical research Institute, Seoul National University Hospital, Seoul, Korea
\end{abstract}

Background: Prader-Willi syndrome (PWS) and Angelman syndrome (AS) are genomic imprinting disorders that are mainly caused by a deletion on 15q11-q13, the uniparental disomy of chromosome 15 , or an imprinting defect. We evaluated the utility of methylation-specific multiplex ligation-dependent probe amplification (MS-MLPA) as a diagnostic tool and for demonstrating the relationship between molecular mechanisms and clinical presentation.

Methods: We performed MS-MLPA using DNA samples from 93 subjects (45 PWS, 24 AS, and 24 non-PWS/AS controls) who had previously undergone MS-PCR for the diagnosis of PWS/AS. We compared the results of both assays, and patients' clinical phenotypes were reviewed retrospectively.

Results: MS-MLPA showed a 100\% concordance rate with MS-PCR. Among the 45 PWS patients, 26 (57.8\%) had a deletion of $15 q 11-q 13$, and the others (42.2\%) had uniparental disomy 15 or an imprinting defect. Among the 24 AS patients, 16 (66.7\%) had a deletion of 15q11-q13, 7 AS patients (29.2\%) had uniparental disomy 15 or an imprinting defect, and one AS patient (4.2\%) showed an imprinting center deletion.

Conclusions: MS-MLPA has clinical utility for the diagnosis of PWS/AS, and it is superior to MS-PCR in that it can identify the molecular mechanism underlying the disease.

Key Words: Prader-Willi syndrome, Angelman syndrome, Methylation-specific multiplex ligation-dependent probe amplification, Methylation-specific PCR, Diagnosis, Utility
Received: August 22, 2020

Revision received: November 17, 2020

Accepted: July 6, 2021

\section{Corresponding author:}

Sung Sup Park, M.D., Ph.D.

Department of Laboratory Medicine, Seoul National University Hospital, 101 Daehakro, Jongno-gu, Seoul 03080, Korea

Tel: $+82-2-2072-3206$

Fax: +82-2-747-0359

E-mail: sparkle@snu.ac.kr

\section{INTRODUCTION}

Prader-Willi syndrome (PWS, OMIM 176270) and Angelman syndrome (AS, OMIM 105830) are caused by the loss of expression of imprinted genes at 15q11-q13 [1]. PWS results from the absence of the paternal allele of 15q11-q13, whereas AS results from the absence of the maternal allele in the same region [2].
This phenomenon is called genomic imprinting. PWS and AS occur in one in 10,000-30,000 live births [3].

Both syndromes are neurodevelopmental disorders; however, their clinical phenotypes differ [4]. PWS is characterized by neonatal hypotonia, feeding problems, failure to thrive, hypogonadism, and childhood-onset obesity [5]. AS patients present with seizures, microcephaly, and severe developmental delay [6]. 
When a neonate shows neonatal hypotonia or developmental delay, PWS or AS should be considered as a part of the differential diagnosis.

Several molecular mechanisms lead to PWS and AS: deletion, uniparental disomy (UPD), imprinting defect (ID), and balanced translocation [7]. Deletion of 15q11-q13 accounts for approximately $70 \%$ of cases and is the leading cause of both syndromes. Deletions are subdivided into typical type I or II deletion, which respectively range from breakpoint (BP)1 to $\mathrm{BP} 3$ or from $\mathrm{BP} 2$ to BP3, and atypical deletion [8]. UPD is mostly due to maternal meiotic non-disjunction and accounts for 3\%-30\% of cases, whereas ID causes 1\%-5\% [9]. Loss of UBE3A function causes AS in 10\%-20\% of patients [10].

Because the molecular mechanisms of PWS and AS determine the recurrence risk, prognosis, and clinical phenotypes, understanding the genetic profiles of these diseases can help clinicians make an accurate diagnosis and counsel patients and their families appropriately [11]. Methylation-specific multiplex ligation-dependent probe amplification (MS-MLPA) is a diagnostic method for the simultaneous detection of copy number abnormalities and methylation status [12]. It can distinguish deletional types from non-deletional types of PWS and AS. We evaluated the clinical utility of MS-MLPA in comparison with that of methylation-specific (MS)-PCR in Korean PWS and AS patients. In addition, we investigated the relationship between clinical phenotypes and molecular mechanisms determined by MS-MLPA.

\section{METHODS}

\section{Patients and samples}

We retrospectively reviewed patients who underwent MS-PCR for PWS and AS in the Seoul National University Hospital (SNUH), Seoul, Korea between March 2007 and July 2018. We selected 45 PWS and 24 AS patients who provided informed consent for

Table 1. Frequency of PWS Holm diagnostic criteria in 45 PWS patients

\begin{tabular}{|c|c|c|}
\hline & Diagnostic criteria & Patients, N (\%) \\
\hline \multicolumn{3}{|l|}{ Major } \\
\hline 1. & Neonatal and infantile central hypotonia with poor suck, gradually improving with age & $36(80.0)$ \\
\hline 2. & Feeding problems in infancy with need for special feeding techniques and poor weight gain/failure to thrive & $31(68.9)$ \\
\hline 3. & $\begin{array}{l}\text { Excessive or rapid weight gain on weight-for-length chart after } 12 \text { months and before the age of six years, central obesity in the absence } \\
\text { of intervention }\end{array}$ & $6(13.3)$ \\
\hline 4. & $\begin{array}{l}\text { Characteristic facial features with dolichocephaly in infancy, narrow face or bifrontal diameter, almond-shaped eyes, small-appearing } \\
\text { mouth with thin upper lip, downturned corners of the mouth }\end{array}$ & $19(42.2)$ \\
\hline 5. & Hypogonadism & $22(48.9)$ \\
\hline 6. & Global developmental delay in a child younger than six years, mild to moderate mental retardation or learning problems in older children & $17(37.8)$ \\
\hline 7. & Hyperphagia/food foraging/obsession with food & $3(6.7)$ \\
\hline 8. & Deletion of 15q11-q13 or other appropriate molecular abnormality in this chromosome region, including maternal disomy & $45(100.0)$ \\
\hline \multicolumn{3}{|c|}{ ( } \\
\hline 1. & Decreased fetal movement or infantile lethargy or weak cry in infancy, improving with age & $25(55.6)$ \\
\hline 2. & Characteristic behavior problems, temper tantrums, violent outbursts, and obsessive/compulsive behavior & $2(4.4)$ \\
\hline 3. & Sleep disturbance or sleep apnea & $0(0.0)$ \\
\hline 4. & Short stature for genetic background by 15 years of age & $2(4.4)$ \\
\hline 5. & Hypopigmentation-fair skin and hair compared with other family members & $12(26.7)$ \\
\hline 6. & Small hands ( $<25$ th percentile) and/or feet $(<10$ th percentile) for height age & $4(8.9)$ \\
\hline 7. & Narrow hands with straight ulnar border & $0(0.0)$ \\
\hline 8. & Eye abnormalities & $0(0.0)$ \\
\hline 9. & Thick, viscous saliva with crusting at corners of the mouth & $0(0.0)$ \\
\hline 10. & Speech articulation defects & $0(0.0)$ \\
\hline 11. & Skin picking & $0(0.0)$ \\
\hline Total & & 45 \\
\hline
\end{tabular}

Adopted from "Prader-Willi syndrome: consensus diagnostic criteria," by Holm VA, et al. 1993 [5].

Abbreviation: PWS, Prader-Willi syndrome. 
secondary utilization and also selected 24 patients who showed negative MS-PCR results and normal karyotypes. The medical records, including diagnosis, chief complaints, laboratory results, and other clinical information, were reviewed retrospectively. For PWS, Holm diagnostic criteria were calculated (Table 1) [5]. AS 1995 diagnostic criteria were applied for the diagnosis of AS (Table 2) [6]. This study was performed in accordance with the Declaration of Helsinki and was approved by the IRB of SNUH
(IRB approval number 1811-075-985).

\section{MS-MLPA}

MS-MLPA was performed using archived genomic DNA and the standard protocol of the SALSA MLPA Probemix ME028-C1 PWS/ AS kit according to the manufacturer's guideline (MRC-Holland, Amsterdam, Netherlands). In brief, 200 ng of genomic DNA was denatured at $98^{\circ} \mathrm{C}$ for five minutes and hybridized with MEO28

Table 2. Frequency of AS diagnostic criteria in 24 AS patients

\begin{tabular}{|c|c|c|}
\hline & Diagnostic criteria & Patients, N (\%) \\
\hline 1. & Normal prenatal and birth history with normal head circumference and absence of major birth defects & $4(16.7)$ \\
\hline 2. & Developmental delay evident by $6-12$ months of age & $8(33.3)$ \\
\hline 3. & Delayed but forward progression of development & $0(0.0)$ \\
\hline 4. & Normal metabolic, hematologic and chemical laboratory profiles & $2(8.3)$ \\
\hline 5. & Structurally normal brain using MRI or CT & $6(25.0)$ \\
\hline \multicolumn{3}{|c|}{ A. Consistent } \\
\hline 1. & Functionally severe developmental delay & $14(58.3)$ \\
\hline 2. & Movement or balance disorder, usually ataxia of gait and/or tremulous movement of limbs & $6(25.0)$ \\
\hline 3. & Behavioral uniqueness & $3(12.5)$ \\
\hline 4. & Speech impairment, none or minimal use of words & $3(12.5)$ \\
\hline \multicolumn{3}{|c|}{ B. Frequent } \\
\hline 1. & Delayed, disproportionate growth in head circumference, usually resulting in microcephaly by two years of age & $3(12.5)$ \\
\hline 2. & Seizures, onset usually before three years of age & $6(25.0)$ \\
\hline 3. & Abnormal EEG, characteristic pattern with large amplitude slow-spike waves, facilitated by eye closure & $3(12.5)$ \\
\hline \multicolumn{3}{|c|}{ C. Associated } \\
\hline 1. & Flat occiput & $0(0.0)$ \\
\hline 2. & Occipital groove & $0(0.0)$ \\
\hline 3. & Protruding tongue & $0(0.0)$ \\
\hline 4. & Tongue thrusting, suck/swallowing disorders & $0(0.0)$ \\
\hline 5. & Feeding problems during infancy & $0(0.0)$ \\
\hline 6. & Prognathia & $0(0.0)$ \\
\hline 7. & Wide mouth, wide-spaced teeth & $0(0.0)$ \\
\hline 8. & Frequent drooling & $0(0.0)$ \\
\hline 9. & Excessive chewing/mouthing behaviors & $0(0.0)$ \\
\hline 10. & Strabismus & $1(4.2)$ \\
\hline 11. & Hypopigmented skin, light hair and eye color & $2(8.3)$ \\
\hline 12. & Hyperactive lower extremity deep tendon reflexes & $0(0.0)$ \\
\hline 13. & Uplifted, flexed arm position especially during ambulation & $0(0.0)$ \\
\hline 14. & Increased sensitivity to heat & $0(0.0)$ \\
\hline 15. & Sleep disturbance & $3(12.5)$ \\
\hline 16. & Attraction to/fascination with water & $0(0.0)$ \\
\hline Total & & 24 \\
\hline
\end{tabular}

Adopted from "Angelman syndrome: consensus for diagnostic criteria," Williams CA, et al. 1995 [6].

Abbreviations: AS, Angelman syndrome; CT, computed tomography; EEG, electroencephalogram; MRI, magnetic resonance imaging. 
probe mix at $60^{\circ} \mathrm{C}$ for 16 hours. The product was aliquoted into two tubes: one for copy number analysis and one for methylation analysis using methylation-sensitive endonuclease. The PCR products were analyzed using an $A B|3130 x|$ capillary sequencer (Applied Biosystems, Foster City, CA, USA) and the data were analyzed using GeneMarker v.1.51 (SoftGenetics, State College, PA, USA). To normalize peak intensities, we used internal control probe normalization, and the intensity ratios of identical probes from the sample were compared with controls.

\section{Statistical analysis}

The concordance rate between MS-PCR and MS-MLPA was calculated using Cohen's kappa coefficient. Continuous variables were compared using Student's t-test and Mann-Whitney U-test. All statistical tests were two-tailed and performed using SPSS version 25.0 (IBM, Armonk, NY, USA). Results were considered statistically significant at $P<0.05$.
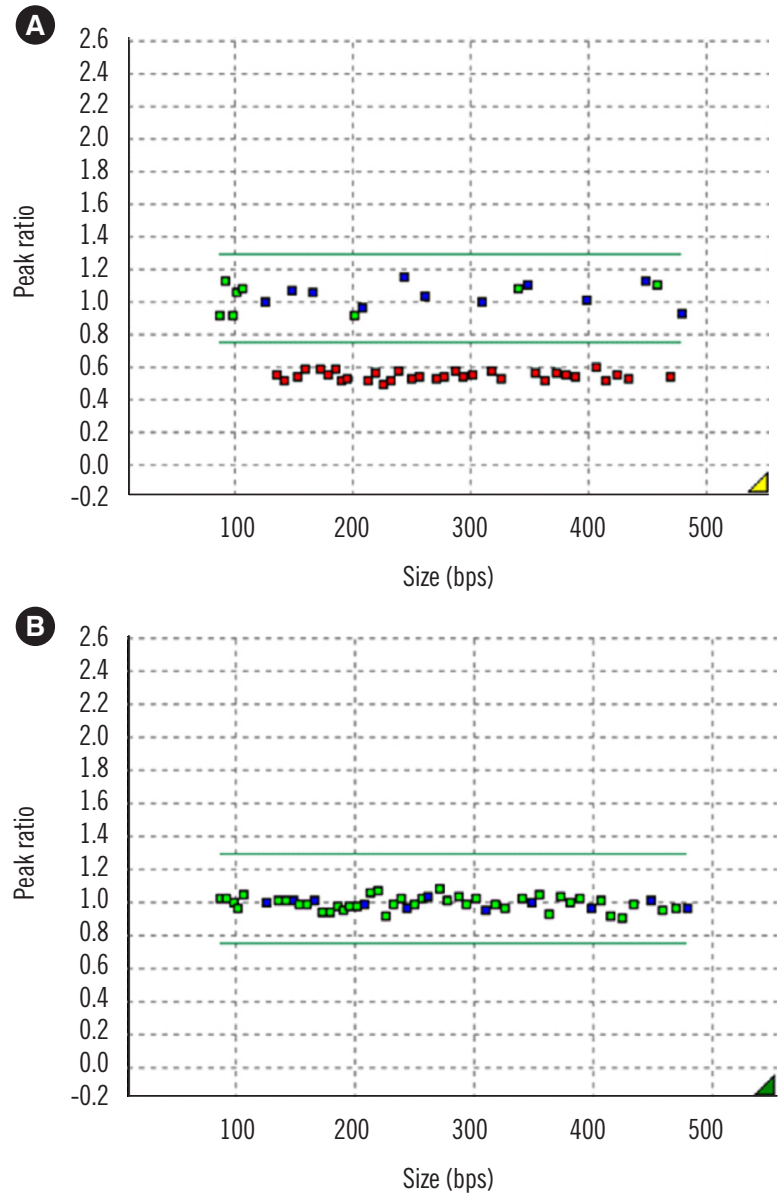

Fig. 1. MS-MLPA results of PWS patients. (A) Deletion type of PWS. digested). Abbreviations: MS-MLPA, methylation-specific multiplex ligation-dependent probe amplification; PWS, Prader-Willi syndrome.

\section{RESULTS}

\section{Comparison of MS-PCR and MS-MLPA}

There were no discordant results between MS-PCR and MSMLPA, with 45 patients diagnosed as having PWS, 24 patients as having AS, and 24 non-PWS/AS controls. Therefore, the concordance rate was $100 \%$, and Cohen's kappa was 1.0, which indicates perfect agreement.

\section{Genetic subtypes of PWS and AS}

Unlike MS-PCR, MS-MLPA could discriminate between the deletion and non-deletion types (Figs. 1 and 2). Among the 45 PWS patients, 26 (57.8\%) had deletions on the q arm of chromosome 15: eight had a type I deletion, 17 had a type II deletion, and one had an atypical deletion (Fig. 3A). The atypical deletion ranged from SNRPN to GABRB3, which is shorter than typical types. Nineteen PWS patients (42.2\%) had UPD/ID. Among the 24 AS
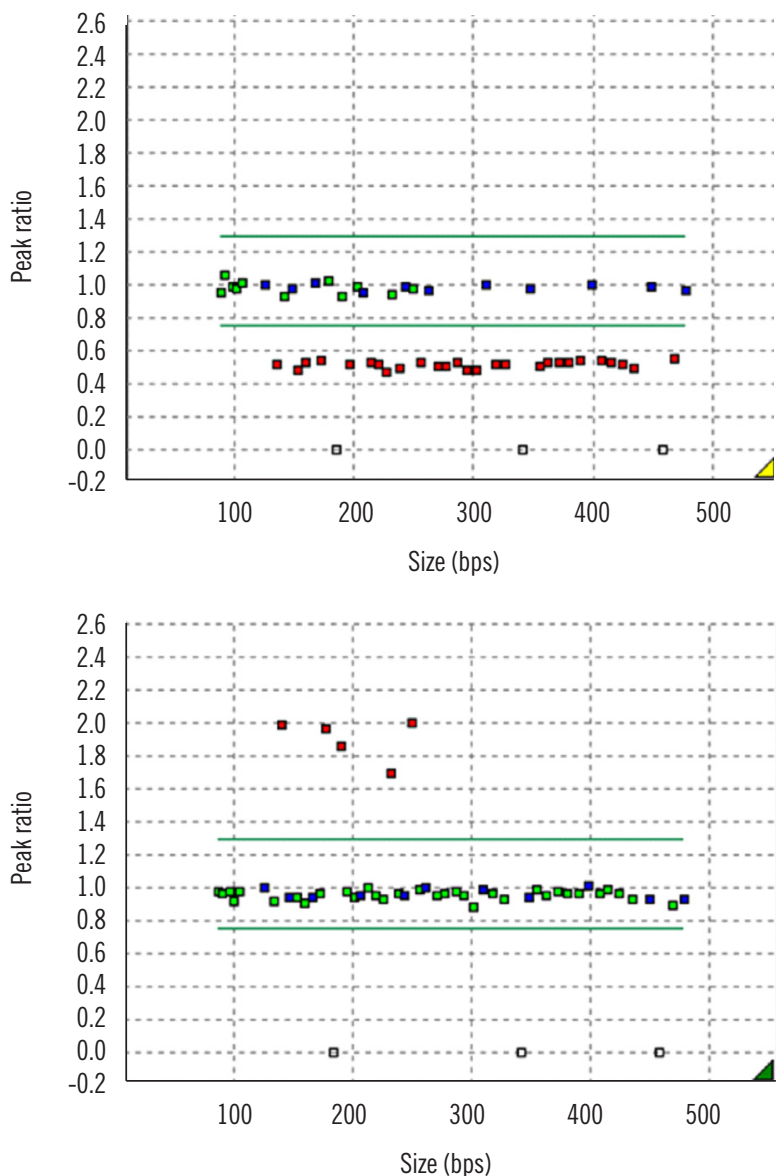
(B) Non-deletion type of PWS. (Left panels: undigested, right panels: 
Kim B, et al.

MS-MLPA for Prader-Willi and Angelman syndromes

patients, 16 (66.7\%) had deletions, seven (29.2\%) had UPD/ ID, and one (4.2\%) showed microdeletion of the AS-shortest region of deletion overlap, which corresponds to an imprinting center (IC) deletion (Fig. 2C). Of the 16 patients with deletions, seven had type I deletions, and nine had type II deletions (Fig. 3B).
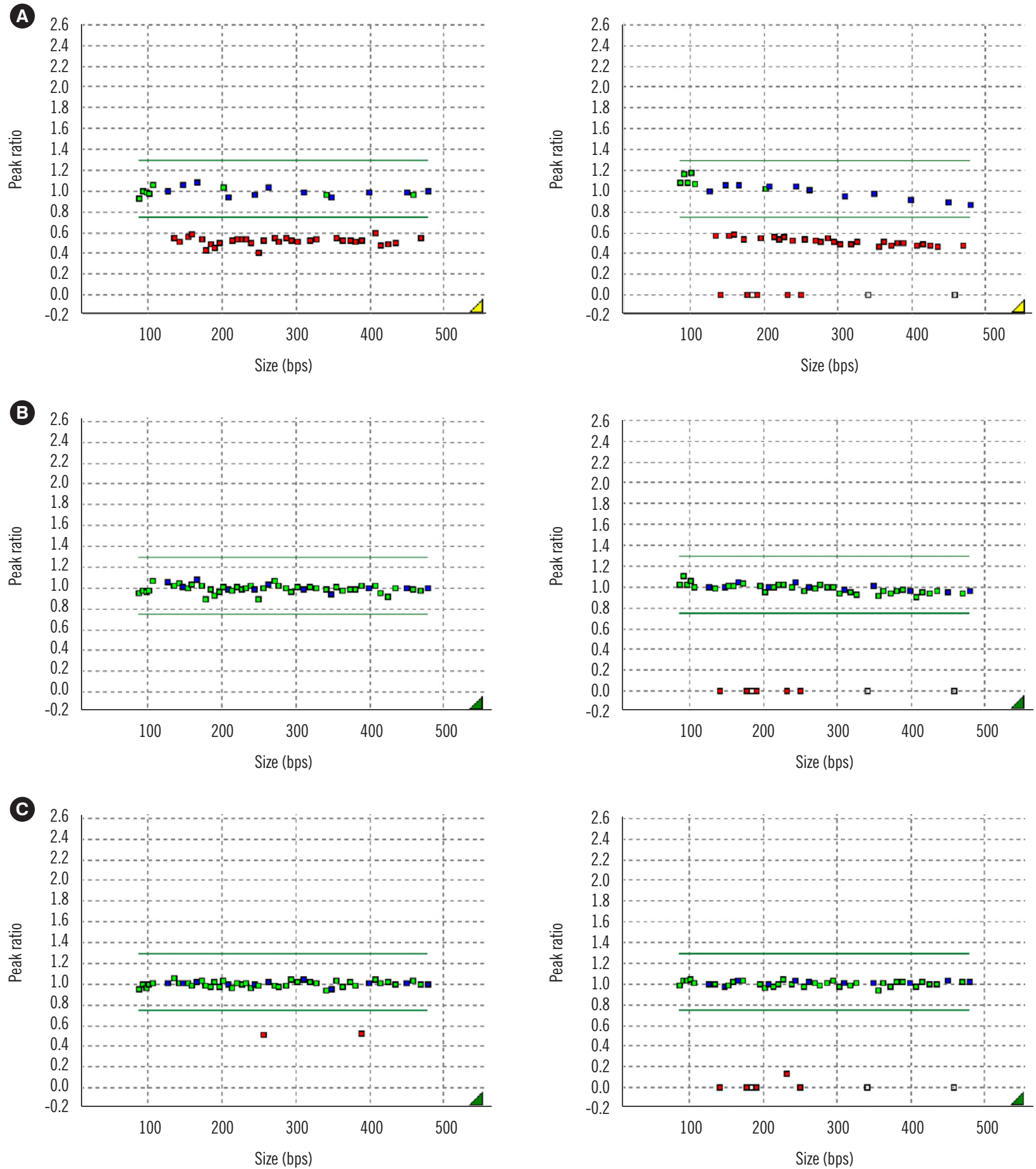

Fig. 2. MS-MLPA results of AS patients. (A) Deletion type of AS. (B) Non-deletion type of AS. (C) IC deletion of AS. (Left panels: undigested, right panels: digested).

Abbreviations: AS, Angelman syndrome; IC, imprinting center; MS-MLPA, methylation-specific multiplex ligation-dependent probe amplification. 

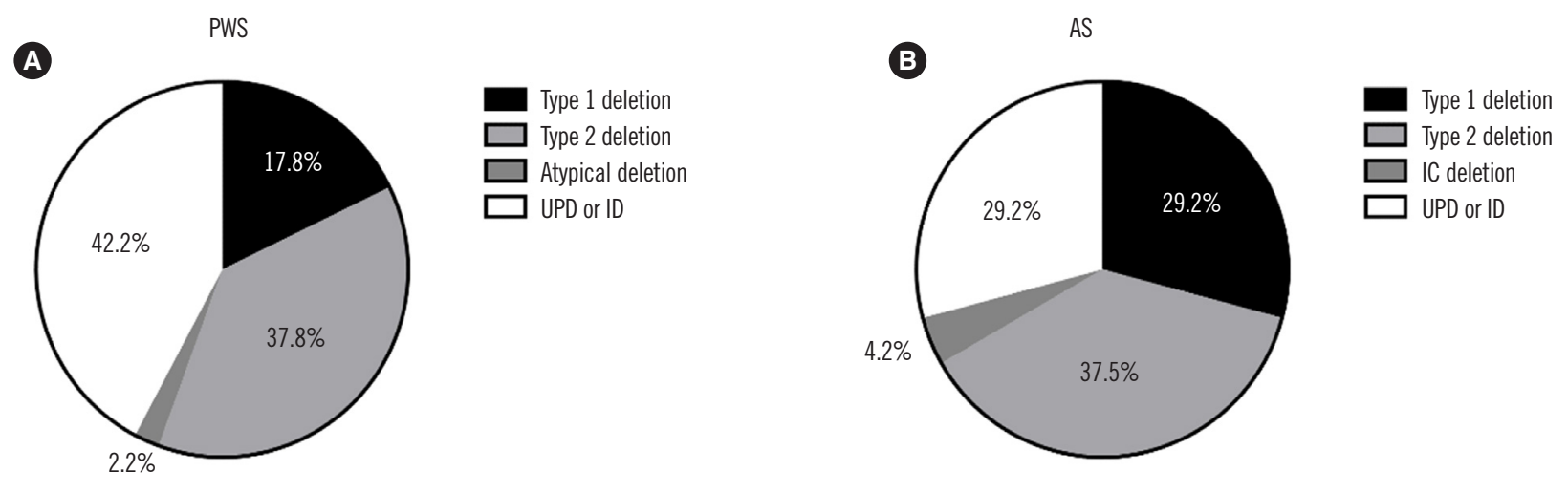

Fig. 3. Distribution of molecular mechanisms in (A) PWS and (B) AS patients.

Abbreviations: AS, Angelman syndrome; IC, imprinting center; ID, imprinting defect; PWS, Prader-Willi syndrome; UPD, uniparental disomy.

\section{Clinical characteristics and molecular mechanisms of PWS and AS}

The male:female ratio of PWS was 1.2:1, and that of AS was 0.6:1 (Table 3). The median age of PWS patients at diagnosis was four months (1-187 months), and that of AS patients was 24.5 months (9-95 months). In AS, the age at diagnosis differed according to the molecular mechanism: patients with the deletion type were diagnosed earlier than those with UPD/ID types (23.8 months vs. 55.7 months, $P=0.002$ ). Birth weight also significantly differed according to the molecular mechanism: patients with the deletion type weighed more at birth than those with the UPD type in PWS and vice versa in AS (2.7 kg vs. 2.4 $\mathrm{kg}, P=0.039$ and $2.9 \mathrm{~kg}$ vs. $3.5 \mathrm{~kg}, P=0.035$ ).

PWS patients mainly showed neonatal hypotonia, developmental delay, altered mentality, failure to thrive, waddling gait, or obesity. Most AS patients (91.7\%) visited our hospital for developmental delay, except two patients who had seizures and torticollis as the main problem. In PWS cases, the mean Holm score was 4.46, which is below the diagnostic criteria (Holm score 6). We observed no notable difference in the Holm scores of PWS according to the molecular mechanism or deletion range.

\section{DISCUSSION}

Chromosomal microarray (CMA) is currently considered the firsttier diagnostic genetic test for neurodevelopmental disorders [14, 15]. However, there remains a necessity for methylation analysis, especially for imprinting disorders, because CMA is not sufficient for diagnosis of these disorders [16]. We showed that MSMLPA can not only diagnose PWS and AS, but also reveal the underlying molecular mechanisms. We also demonstrated the relationship between molecular mechanisms and clinical characteristics of Korean PWS and AS patients. Our findings support that MS-MLPA is a useful diagnostic test for PWS and AS.

We detected the deletion type in $57.8 \%$ of PWS cases, which is in contrast to results in a previous Korean study in 2004, in which deletion accounted for $80 \%$ of PWS cases [17]. In line with our results, recent studies have demonstrated that the deletion type in PWS constitutes approximately $60 \%$ of total cases [18, 19]. Butler, et al. [18] reported that in PWS, the maternal age was higher in UPD cases than in deletion cases. Although we were not able to assess maternal age for all patients, the average maternal age in Korea is increasing yearly, from 30.0 years in 2004 to 32.4 years in 2016, according to the Korea Statistics [20]. Thus, the increase in the non-deletion type may be because UPD is more prone to occur as maternal age increases.

PWS patients with type I deletions show a more severe phenotype than those with type II deletions [21]; however, there were no significant differences in the diagnostic scores of our patients according to the deletion range. The mean Holm score of total PWS was even lower than the diagnostic score. This may be explained by poor clinical evaluation or diagnosis before clinical symptoms present due to advanced molecular diagnosis.

An atypical deletion, ranging from SNRPN to GABRB3, was detected in one PWS patient (case 39). Additional CMA results confirmed that the patient had a $2.8 \mathrm{Mb}$ deletion from PWRN2 to GABRB3. He visited our hospital for lymph node enlargement at 12 years of age. At that time, he had mild learning disabilities and obesity $[22,23]$. His Holm score was 5, which is higher than the mean score of PWS patients. This deletion type showed that MKRN3, MAGEL2, NDN, and C15orf2 are not indispensable for PWS symptoms [24].

One AS patient with an IC deletion (case 66) visited our hospital for developmental delay at 36 months of age. He showed a facial dysmorphism, developmental delay and generalized seizures, which indicated a diagnosis of AS. Although the probabil- 
Kim B, et al.

MS-MLPA for Prader-Willi and Angelman syndromes

Table 3. Clinical features and molecular mechanisms in PWS and AS patients

\begin{tabular}{|c|c|c|c|c|c|c|}
\hline Case number & Sex & $\begin{array}{l}\text { Age at diagnosis } \\
\text { (month) }\end{array}$ & $\begin{array}{l}\text { Birth weight } \\
(\mathrm{kg})\end{array}$ & MS-PCR & MS-MLPA & Clinical features \\
\hline 1 & $\mathrm{~F}$ & 13 & 3.4 & PWS & Deletion (Type II) & Neonatal hypotonia \\
\hline 2 & M & 2 & 2.6 & PWS & Deletion (Type II) & Neonatal hypotonia \\
\hline 3 & M & 2 & 3.1 & PWS & Deletion (Type II) & Neonatal hypotonia \\
\hline 4 & M & 40 & 2.4 & PWS & Deletion (Type I) & Developmental delay \\
\hline 5 & $\mathrm{~F}$ & 6 & 3.0 & PWS & Deletion (Type II) & Developmental delay \\
\hline 6 & $\mathrm{~F}$ & 1 & 2.8 & PWS & Deletion (Type II) & Altered mentality \\
\hline 7 & $\mathrm{~F}$ & 1 & 2.6 & PWS & Deletion (Type II) & Neonatal hypotonia \\
\hline 8 & $\mathrm{~F}$ & 25 & 3.3 & PWS & UPD/ID & Developmental delay \\
\hline 9 & M & 35 & 1.9 & PWS & UPD/ID & Developmental delay \\
\hline 10 & M & 87 & 2.0 & PWS & Deletion (Type II) & Waddling gait \\
\hline 11 & $\mathrm{~F}$ & 4 & 2.9 & PWS & Deletion (Type I) & Developmental delay \\
\hline 12 & M & 1 & 3.1 & PWS & UPD/ID & Neonatal hypotonia \\
\hline 13 & M & 3 & 2.2 & PWS & UPD/ID & Neonatal hypotonia \\
\hline 14 & M & 6 & 2.7 & PWS & Deletion (Type II) & Neonatal hypotonia \\
\hline 15 & M & 11 & 2.8 & PWS & UPD/ID & Neonatal hypotonia \\
\hline 16 & M & 1 & 2.5 & PWS & Deletion (Type II) & Poor sucking \\
\hline 17 & $\mathrm{~F}$ & 1 & 2.8 & PWS & Deletion (Type I) & Poor sucking \\
\hline 18 & M & 123 & 2.2 & PWS & UPD/ID & Developmental delay \\
\hline 19 & M & 4 & 2.8 & PWS & Deletion (Type II) & Neonatal hypotonia \\
\hline 20 & $\mathrm{~F}$ & 5 & 3.2 & PWS & UPD/ID & Neonatal hypotonia \\
\hline 21 & $\mathrm{~F}$ & 2 & 2.6 & PWS & Deletion (Type I) & Developmental delay \\
\hline 22 & M & 187 & 2.2 & PWS & UPD/ID & Developmental delay \\
\hline 23 & M & 1 & 2.9 & PWS & Deletion (Type I) & Neonatal hypotonia \\
\hline 24 & $\mathrm{~F}$ & 5 & 2.3 & PWS & UPD/ID & Developmental delay \\
\hline 25 & M & 4 & 3.1 & PWS & UPD/ID & Fever \\
\hline 26 & $\mathrm{~F}$ & 1 & 2.6 & PWS & Deletion (Type II) & Neonatal hypotonia \\
\hline 27 & M & 1 & 2.6 & PWS & Deletion (Type I) & Neonatal hypotonia \\
\hline 28 & M & 26 & 2.3 & PWS & UPD/ID & Developmental delay \\
\hline 29 & $\mathrm{~F}$ & 114 & 2.2 & PWS & UPD/ID & Obesity \\
\hline 30 & M & 144 & 2.8 & PWS & Deletion (Type II) & Obesity \\
\hline 31 & M & 50 & 0.7 & PWS & UPD/ID & Failure to thrive \\
\hline 32 & $\mathrm{~F}$ & 4 & 2.8 & PWS & Deletion (Type II) & Developmental delay \\
\hline 33 & M & 2 & 2.4 & PWS & Deletion (Type II) & Neonatal hypotonia \\
\hline 34 & $\mathrm{~F}$ & 5 & 1.5 & PWS & UPD/ID & Developmental delay \\
\hline 35 & M & 2 & 3.0 & PWS & UPD/ID & Neonatal hypotonia \\
\hline 36 & M & 2 & 2.2 & PWS & Deletion (Type II) & Neonatal hypotonia \\
\hline 37 & $\mathrm{~F}$ & 1 & 2.7 & PWS & Deletion (Type I) & Neonatal hypotonia \\
\hline 38 & $\mathrm{~F}$ & 10 & 3.2 & PWS & Deletion (Type II) & Developmental delay \\
\hline 39 & M & 150 & 3.6 & PWS & Deletion (Atypical) & Lymphadenopathy \\
\hline 40 & $\mathrm{~F}$ & 3 & 1.9 & PWS & UPD/ID & Neonatal hypotonia \\
\hline
\end{tabular}


Table 3. Continued

\begin{tabular}{|c|c|c|c|c|c|c|}
\hline Case number & Sex & $\begin{array}{l}\text { Age at diagnosis } \\
\text { (month) }\end{array}$ & $\begin{array}{l}\text { Birth weight } \\
\quad(\mathrm{kg})\end{array}$ & MS-PCR & MS-MLPA & Clinical features \\
\hline 41 & M & 1 & 2.4 & PWS & Deletion (Type I) & Neonatal hypotonia \\
\hline 42 & $\mathrm{~F}$ & 5 & 2.6 & PWS & UPD/ID & Neonatal hypotonia \\
\hline 43 & $\mathrm{~F}$ & 155 & 2.3 & PWS & UPD/ID & Headache \\
\hline 44 & $\mathrm{~F}$ & 7 & 2.6 & PWS & UPD/ID & Developmental delay \\
\hline 45 & M & 2 & 2.8 & PWS & Deletion (Type II) & Neonatal hypotonia \\
\hline 46 & M & 21 & 3.4 & AS & Deletion (Type II) & Developmental delay \\
\hline 47 & M & 79 & 3.0 & AS & Deletion (Type I) & Developmental delay \\
\hline 48 & $\mathrm{~F}$ & 36 & $\mathrm{~N} / \mathrm{A}$ & AS & UPD/ID & Developmental delay \\
\hline 49 & $\mathrm{~F}$ & 56 & 2.5 & AS & Deletion (Type I) & Developmental delay \\
\hline 50 & M & 14 & 2.9 & AS & Deletion (Type I) & Developmental delay \\
\hline 51 & $\mathrm{~F}$ & 34 & $\mathrm{~N} / \mathrm{A}$ & AS & UPD/ID & Developmental delay \\
\hline 52 & M & 63 & 2.7 & AS & UPD/ID & Seizures \\
\hline 53 & $\mathrm{~F}$ & 25 & 2.4 & AS & Deletion (Type I) & Developmental delay \\
\hline 54 & $\mathrm{~F}$ & 12 & 3.0 & AS & Deletion (Type I) & Developmental delay \\
\hline 55 & $\mathrm{~F}$ & 16 & $\mathrm{~N} / \mathrm{A}$ & AS & Deletion (Type I) & Developmental delay \\
\hline 56 & $\mathrm{~F}$ & 11 & 2.6 & AS & Deletion (Type II) & Developmental delay \\
\hline 57 & $\mathrm{~F}$ & 17 & 2.6 & AS & Deletion (Type II) & Developmental delay \\
\hline 58 & $\mathrm{~F}$ & 9 & 2.5 & AS & Deletion (Type II) & Torticollis \\
\hline 59 & $\mathrm{~F}$ & 17 & 2.6 & AS & Deletion (Type II) & Developmental delay \\
\hline 60 & $\mathrm{~F}$ & 13 & 4.0 & AS & Deletion (Type II) & Developmental delay \\
\hline 61 & $\mathrm{~F}$ & 29 & 2.3 & AS & Deletion (Type II) & Developmental delay \\
\hline 62 & $\mathrm{~F}$ & 19 & 3.2 & AS & Deletion (Type I) & Developmental delay \\
\hline 63 & $\mathrm{~F}$ & 19 & 3.1 & AS & Deletion (Type II) & Developmental delay \\
\hline 64 & M & 95 & 3.7 & AS & UPD/ID & Developmental delay \\
\hline 65 & $\mathrm{~F}$ & 31 & 3.3 & AS & UPD/ID & Developmental delay \\
\hline 66 & M & 38 & 3.0 & AS & IC deletion & Developmental delay \\
\hline 67 & M & 76 & 4.6 & AS & UPD/ID & Developmental delas \\
\hline 68 & M & 55 & 3.2 & AS & UPD/ID & Developmental delay \\
\hline 69 & M & 24 & $\mathrm{~N} / \mathrm{A}$ & AS & Deletion (Type II) & Developmental delay \\
\hline
\end{tabular}

Abbreviations: AS, Angelman syndrome; F, female; IC, imprinting center; ID, imprinting defect; M, male; MS-MLPA, methylation-specific multiplex ligationdependent probe amplification; MS-PCR, methylation-specific PCR; N/A, not available; PWS, Prader-Willi syndrome; UPD, uniparental disomy.

ity of recurrence of IC is up to $50 \%$, which is the highest recurrence rate for PWS/AS, his younger sister, who is the secondborn child, is healthy [25].

To the best of our knowledge, this study is the largest cohort covering both PWS and AS, with clinical information. A few previous studies dealt with the molecular diagnosis of these syndromes, but most of them focused only on PWS or presented no clinical findings $[2,26]$.

Our study has some limitations. First, there is an intrinsic limitation to MS-MLPA in that it cannot distinguish UPD from ID. To distinguish these two mechanisms, microsatellite analysis or single nucleotide variant analysis is required [27]. However, we successfully differentiated the IC deletion type, which has a $50 \%$ recurrence risk, using MS-MLPA. In addition, we could not evaluate the behavioral or psychological status of the patients due to the lack of such information in the medical records. Lastly, this was a retrospective and single-center study. Thus, a selection bias may exist, and further large-scale prospective studies for PWS and AS are needed.

AS caused by a UBE3A variant, which accounts for $20 \%$ of 
AS cases, cannot be diagnosed using MS-MLPA, and further diagnostic testing is needed. To date, only two patients have been diagnosed as having AS due to UBE3A pathogenic variants in our laboratory. Therefore, we propose the use of MS-MLPA as a first-line diagnostic tool for PWS or AS, in accordance with the EMQN/ACGS guidelines [28]. The next step would be UBE3A variant analysis of samples from patients who are highly suspected of having AS, as well as maternal samples.

In conclusion, MS-PCR and MS-MLPA show perfect diagnostic concordance, and MS-MLPA can substitute for MS-PCR. In addition, MS-MLPA provides more information about the molecular mechanisms underlying the diseases and may be a helpful tool for genetic counseling of families with PWS and AS. Finally, patients who are strongly suspected of having AS and show negative MS-MLPA results should undergo additional testing.

\section{ACKNOWLEDGEMENTS}

None.

\section{AUTHOR CONTRIBUTIONS}

Kim B interpreted and statistically analyzed data and wrote the main manuscript; Park $Y$ and Cho SI performed the experiments and filled out the clinical research form. Kim MJ, Chae JH, Kim JY, and Seong MW participated in study design and reviewed the final manuscript; Park SS conceived the study and study design and reviewed and approved the final manuscript. All authors have read and approved the manuscript.

\section{CONFLICTS OF INTEREST}

None declared.

\section{RESEARCH FUNDING}

None declared.

\section{ORCID}

\author{
Boram Kim \\ Yongsook Park \\ Sung Im Cho \\ Man Jin Kim \\ Jong-Hee Chae \\ Ji Yeon Kim
}

Moon-Woo Seong

Sung Sup Park

https://orcid.org/0000-0003-2954-3677

https://orcid.org/0000-0003-3754-4848

\section{REFERENCES}

1. Õiglane-Shlik E, Talvik T, Zordania R, Põder H, Kahre T, Raukas E, et al. Prevalence of Angelman syndrome and Prader-Willi syndrome in Estonian children: sister syndromes not equally represented. Am J Med Genet A 2006;140:1936-43.

2. Botezatu A, Puiu M, Cucu N, Diaconu CC, Badiu C, Arsene C, et al. Comparative molecular approaches in Prader-Willi syndrome diagnosis. Gene 2016;575:353-8.

3. Ferreira IR, Costa RA, Gomes LHF, Dos Santos Cunha WD, Tyszler LS, Freitas $\mathrm{S}$, et al. A newborn screening pilot study using methylation-sensitive high resolution melting on dried blood spots to detect Prader-Willi and Angelman syndromes. Sci Rep 2020;10:13026.

4. Ehrhart F, Janssen KJM, Coort SL, Evelo CT, Curfs LMG. Prader-Willi syndrome and Angelman syndrome: visualisation of the molecular pathways for two chromosomal disorders. World J Biol Psychiatry 2019;20: 670-82.

5. Holm VA, Cassidy SB, Butler MG, Hanchett JM, Greenswag LR, Whitman BY, et al. Prader-Willi syndrome: consensus diagnostic criteria. Pediatrics 1993;91:398-402.

6. Williams CA, Angelman H, Clayton-Smith J, Driscoll DJ, Hendrickson $\mathrm{JE}$, Knoll JH, et al. Angelman syndrome: consensus for diagnostic criteria. Am J Med Genet 1995;56:237-8.

7. Nicholls RD, Saitoh S, Horsthemke B. Imprinting in Prader-Willi and Angelman syndromes. Trends Genet 1998;14:194-200.

8. Butler MG, Miller JL, Forster JL. Prader-Willi syndrome - clinical genetics, diagnosis and treatment approaches: an update. Curr Pediatr Rev 2019;15:207-44.

9. Buiting K. Prader-Willi syndrome and Angelman syndrome. Am J Med Genet C Semin Med Genet 2010;154C:365-76.

10. Ramsden SC, Clayton-Smith J, Birch R, Buiting K. Practice guidelines for the molecular analysis of Prader-Willi and Angelman syndromes. BMC Med Genet 2010;11:70.

11. Smith A and Hung D. The dilemma of diagnostic testing for Prader-Willi syndrome. Transl Pediatr 2017;6:46-56.

12. Nygren AO, Ameziane N, Duarte HM, Vijzelaar RN, Waisfisz Q, Hess CJ, et al. Methylation-specific MLPA (MS-MLPA): simultaneous detection of $\mathrm{CpG}$ methylation and copy number changes of up to 40 sequences. Nucleic Acids Res 2005;33:e128.

13. Kubota T, Das S, Christian SL, Baylin SB, Herman JG, Ledbetter DH. Methylation-specific PCR simplifies imprinting analysis. Nat Genet 1997; 16:16-7.

14. Shin S, Yu N, Choi JR, Jeong S, Lee KA. Routine chromosomal microarray analysis is necessary in Korean patients with unexplained developmental delay/mental retardation/autism spectrum disorder. Ann Lab Med 2015;35:510-8.

15. Jang W, Kim Y, Han E, Park J, Chae H, Kwon A, et al. Chromosomal microarray analysis as a first-tier clinical diagnostic test in patients with developmental delay/intellectual disability, autism spectrum disorders, and multiple congenital anomalies: a prospective multicenter study in Korea. Ann Lab Med 2019;39:299-310.

16. Liu S, Zhang K, Song F, Yang Y, Lv Y, Gao M, et al. Uniparental disomy of chromosome 15 in two cases by chromosome microarray: a lesson worth thinking. Cytogenet Genome Res 2017;152:1-8.

17. Kim HJ, Cho HJ, Jin DK, Kwon EK, Ki CS, Kim JW, et al. Genetic basis 
of Prader-Willi syndrome in Korea: less uniparental disomy than has been recognized? Clin Genet 2004;66:368-72.

18. Butler MG, Hartin SN, Hossain WA, Manzardo AM, Kimonis V, Dykens E, et al. Molecular genetic classification in Prader-Willi syndrome: a multisite cohort study. J Med Genet 2019;56:149-53.

19. Singh P, Mahmoud R, Gold JA, Miller JL, Roof E, Tamura R, et al. Multicentre study of maternal and neonatal outcomes in individuals with Prader-Willi syndrome. J Med Genet 2018;55:594-8.

20. Kim HE, Song IG, Chung SH, Choi YS, Bae CW. Trends in birth weight and the incidence of low birth weight and advanced maternal age in Korea between 1993 and 2016. J Korean Med Sci 2019;34:e34.

21. Butler MG, Bittel DC, Kibiryeva N, Talebizadeh Z, Thompson T. Behavioral differences among subjects with Prader-Willi syndrome and type I or type II deletion and maternal disomy. Pediatrics 2004;113:565-73.

22. Kim SJ, Miller JL, Kuipers PJ, German JR, Beaudet AL, Sahoo T, et al. Unique and atypical deletions in Prader-Willi syndrome reveal distinct phenotypes. Eur J Hum Genet 2012;20:283-90.

23. Hassan M and Butler MG. Prader-Willi syndrome and atypical submicroscopic 15q11-q13 deletions with or without imprinting defects. Eur J
Med Genet 2016;59:584-9.

24. Kanber D, Giltay J, Wieczorek D, Zogel C, Hochstenbach R, Caliebe A, et al. A paternal deletion of MKRN3, MAGEL2 and NDN does not result in Prader-Willi syndrome. Eur J Hum Genet 2009;17:582-90.

25. Hartin SN, Hossain WA, Weisensel N, Butler MG. Three siblings with Prader-Willi syndrome caused by imprinting center microdeletions and review. Am J Med Genet A 2018;176:886-95.

26. Lu W, Qi Y, Cui B, Chen XL, Wu BB, Chen C, et al. Clinical and genetic features of Prader-Willi syndrome in China. Eur J Pediatr 2014;173:816.

27. Wang W, Law HY, Chong SS. Detection and discrimination between deletional and non-deletional Prader-Willi and Angelman syndromes by methylation-specific PCR and quantitative melting curve analysis. J Mol Diagn 2009;11:446-9.

28. Beygo J, Buiting K, Ramsden SC, Ellis R, Clayton-Smith J, Kanber D. Update of the EMQN/ACGS best practice guidelines for molecular analysis of Prader-Willi and Angelman syndromes. Eur J Hum Genet 2019; 27:1326-40. 